stent insertion; $17 \%$ (8 of 46) versus $40 \%$ (20 of 50 ), $p=0.01$.

Conclusions Overall, our results offer quality assurance for our local practice with PLBD use allowing duct clearance and complication rates comparable with published literature (75.5$100 \%$ and $2.6-7.7 \%$, respectively). They also suggest that, in a DGH setting, PLBD can be performed safely and, given the improved duct clearance rates, should be utilised.

\section{PTH-058 ATTEMPTING TOTAL COLONOSCOPY IN INADEQUATELY PREPARED BOWEL: THE DEVIL IS IN THE DETAIL!}

Konstantinos Argyriou, Kristel Yap, Shin Ho, Chi wing Chow, Fateen Waleed, Shiv Budihal, Ana Garcia Marin, Mileidis San Juan-Acosta, Krish Ragunath, Adolfo Parra-Blanco* Nottingham Digestive Diseases Centre, University of Nottingham, NIHR BRC at Nottingham University Hospitals and University of Nottingham, Nottingham, UK

\subsection{6/gutjnl-2019-BSGAbstracts.83}

Introduction Bowel preparation (BP) is a fundament for highquality colonoscopy. Current guidelines suggest repeating colonoscopy within 1 year in case of inadequate bowel preparation (IBP). Up to $83 \%$ of individuals does not repeat the examination, with cecal intubation (CI) . Concurrently, in UK, endoscopists attempt full colonoscopy even if the BP is inadequate, so as to avoid being penalized with low CI rate. The implications of this practice remain unknown.

Methods In a cohort of outpatients with IBP, we aimed to investigate the effect of CI at index colonoscopy on early repeat the examination, and to describe the implications on detecting colorectal neoplasia. Over a 2-year period (20132014), all outpatients who underwent colonoscopy in our center IBP, were identified. Endoscopic records were reviewed as per BP quality, limit of examination, timing between colonoscopies as well as the identification of polyps (size, type, number) and cancer for a minimum 3-year period. BP quality was dichotomized as adequate or inadequate as per the 2012 U.S. Multi-Society Task Force definition for IBP. Multivariate analysis performed to identify factors associated with early repeat colonoscopy.

Results Of 12.948 colonoscopies, IBP was identified in 944. Over the follow-up period, colonoscopy was repeated in 396 (41.9\%) individuals. The mean time (standard deviation) interval between index and repeat colonoscopy was 412 (517) days. At index colonoscopy, CI was achieved in 654 cases (69.6\%). Adenomas (A) were identified in 128 cases advanced neoplasia [62 advanced adenomas (AA)and 41 cancers (c)]. Being on a screening/surveillance program [sig: .00, $\operatorname{Exp}(\mathrm{B})$ : .34 , 95\% C.I.: .24-.48)] and reaching the caecum at index procedure were negatively associated with early repeating colonoscopy. [sig: .00, $\operatorname{Exp}(\mathrm{B}): .46,95 \%$ C.I.: .34-.62)] Among outpatients CI at index examination, 253 had a repeat colonoscopy. $125 \mathrm{~A}, 17 \mathrm{AA}$ and $2 \mathrm{c}$ were only seen on the second examination, corresponding to a miss rate of $38.8 \%, 20.5 \%$ and $8.33 \%$ respectively.

Conclusion 2 out of 5 outpatients with IBP colonoscopy within 3 years. Miss rates for neoplasia is high for colonoscopies with IBP. CI is an independent predictor for not repeating coloscopy in the subsequent 3 -year period. Considering that endoscopists are urged to reach the caecum in each colonoscopy, guidelines need to define how to deal with the cases of IBP, so as to ensure high-quality examinations and an early repeat colonoscopy.

\section{PTH-059 CIRCUMFERENTIAL ESD FOR SQUAMOUS OESOPHAGEAL NEOPLASIA USING THE DOUBLE TUNNEL TECHNIQUE: AN ORGAN PRESERVING PROCEDURE}

${ }^{1}$ Adolfo Parra-Blanco*, 'Miguel Fraile-Lopez, ${ }^{1}$ Jacobo Ortiz Fernandez-Sordo, ${ }^{2}$ Philip Kaye, ${ }^{1}$ Krish Ragunath. ${ }^{1}$ Nottingham Digestive Diseases Centre, University of Nottingham, UK NIHR BRC at Nottingham University Hospitals and University of Nottingham, Nottingham, UK; ${ }^{2}$ Department of Histopathology and Nottingham Digestive Diseases BRC, Nottingham University Hospitals, Nottingham, UK

\subsection{6/gutjnl-2019-BSGAbstracts.84}

Introduction Endoscopic Submucosal Dissection (ESD) is the technique of choice for superficial squamous neoplasia. If the lesion is extensive, circumferential ESD may be needed, and this is more technically challenging. Single tunnel technique has been reported. However, dissecting the submucosal tissue after having completed the tunnel may still be difficult, and traction with the clip-line method has been reported in this scenario. In this video abstract we describe for the first time the double-tunnel technique, with clip-band line traction in a large circumferential oesophageal ESD.

Methods A 48 year old patient, with a personal history of alcohol excess, underwent gastroscopy for dyspepsia and dysphagia, which revealed an early squamous neoplasia proximal to a short segment of Barrett's, as described below., Biopsy revealed high grade dysplasia within the squamous neoplasia. He underwent ESD under General anaesthetic.

With white light endoscopy the lesion only showed some erythema; with Narrow Band Imaging and lugol, it was Paris 0 -IIa+IIb and, size $70 \mathrm{~mm}$. Magnification X 100 showed IPCL type IV, B-1. The planned resection included ESD from 25 to $35 \mathrm{~cm}$ from the incisors.

The equipment used was a Olympus GIFH260Z gastroscope, an ERBE VIO 200D electrosurgical unit, CO2 for insufflation, a short overtube (Sumius, Sumitomo) and injection solution was Gelofusin with adrenaline and indigo carmine. The technique for ESD included: 1) marking oral and anal side, 2) injection and circumferential incision at the distal end with a Dual knife jet (DK-J), 3) first tunnel at 5 o'clock, with DK-J, 4) second tunnel at 11 o'clock with DK-J, 4) connecting the pillars between both tunnels with DK-J, IT knife nano and SB knife junior. Traction was applied by the ClipBand line technique, in order to facilitate the dissection between the pillars. The whole procedure time from the initial cut to the end of the dissection was 287 minutes. One clip was applied on a small non-transmural injury of the muscle layer. Visible vessels were coagulated with a bipolar haemostatic forceps.

Oral steroid (prednisolone $30 \mathrm{mg}$ po od) was prescribed on tapering schedule for 6 weeks. A follow up endoscopy 8 days after the procedure showed lumen patency without stenosis.

Results Histopathological analysis revealed high grade dysplasia amounting to carcinoma in situ, which was completely excised. No evidence of lymphovascular invasion noticed.

Conclusions In conclusion, double-tunnel dissection technique with a combination of knives allows safe resection of large superficial oesophageal neoplasia, even if these are circumferential. The Clip-band line traction method further facilitates this technique. 